\title{
physiokongress: Physiotherapie bewegt sich
}

Drei Tage Physiotherapie pur und vieles mehr bot der physiokongress in Aachen. Die Besucher tauschten sich rege untereinander aus, informierten sich über aktuelle Entwicklungen in der Physiotherapie und sammelten wertvolle Impulse für ihren Alltag. Es zeigte sich, Physiotherapeuten sind nicht nur Experten der Bewegung, sie setzen auch einiges in Bewegung.

Ü ber 1.200 Besucher kamen vom 17. bis 19. Mai nach Aachen zum 3. physiokongress (॰ Abb. 1). Sie lockte nicht nur der Austausch mit Kollegen und Neuigkeiten aus der „Physiowelt“, sondern auch das vielfältige Programm mit nationalen und internationalen Koryphäen. „Wir wollen unserer Berufsgruppe ein Podium geben, auf dem man sich fachlich, interdisziplinär und berufspolitisch austauschen kann “, beschreibt Physiotherapeutin Rosi Haarer-Becker die Idee, die hinter dem physiokongress steckt und auch gelungen war. Sie plante zusammen mit Uwe Harste, ebenfalls Physiotherapeut, das Programm des diesjährigen Kongresses (॰ Abb. 2 a und b). Es konnte sich sehen lassen! Neben den Schwerpunktthemen Multiple Sklerose, Osteopathie, Schmerz, Sport und Physiotherapie erhielten die Besucher beispielsweise auch zu Themen wie „Wissenschaftlich denken und handeln“ sowie „Un-
Gehirns. Beim Wiederholen von Bewegungen verstärkt sich die Repräsentation des Körperteils auf dem motorischen Kortex im gleichen Maße, wie sie sich auch zum Beispiel nach einer Phase der Immobilisation zurückbildet. Allein das Denken an eine Bewegung aktiviert 15\% der Neuronen im präzentralen Kortex. Diese Erkenntnis ist nicht nur für den Profisport interessant, sondern zum Beispiel auch für die physiotherapeutische Behandlung von Patienten nach Schlaganfall. „Bewegung ist eine Information für das Gehirn, die, wenn sie in einen Kontext eingebunden ist, zum motorischen Lernen führt“, sagte Professor Theo Mulder. Zur Veranschaulichung zeigte der Neuropsychologe eine Videosequenz, die den Behandlungsverlauf eines schwer an Parkinson erkrankten Patienten dokumentierte. Zu sehen war ein alter Herr, der unkoordiniert und wackelig durch den Raum ging. Doch gab man ihm einen Tisch- gungswissenschaftlerin und Dozentin der $\mathrm{FH}$ Hildesheim Annette Probst ( $\odot$ Abb. 4) dem zentralen Thema Bewegung in der Physiotherapie. Die Physiotherapeutin stellte in ihrem Vortrag zunächst Theoriemodelle vor, mit denen Physiotherapeuten heute bereits arbeiten. Sie selbst hat nun zusammen mit ihren Studierenden an der FH Hildesheim ein weiteres Modell entwickelt. Gegenstand dieses Modells ist der Bewegungsbegriff in der Physiotherapie. Bewegung ist ein zentrales Element der Physiotherapie, doch wird sie nicht exklusiv von Physiotherapeuten genutzt. Was macht nun Bewegung in der Physiotherapie aus? Unter dieser Fragestellung entwickelte Probst das Modell. „Das Phänomen Bewegung sollte mit Blick auf seine physiotherapeutische Ausprägung definierbar (...) und im Praxishandeln erkennbar sein“, sagte Probst. Um zu veranschaulichen, wie Bewegung als Bewegungshandlung verstanden werden kann, zeigte sie eine Videosequenz aus dem Film „I, Robot“. In den gezeigten Szenen lernt ein Roboter die soziale Dimension der Bewegung „das Augenzwinkern“ kennen und einzusetzen. Dieser Ausschnitt verdeutlichte die einzelnen Elemente, aus der eine Bewegungshandlung besteht:
8

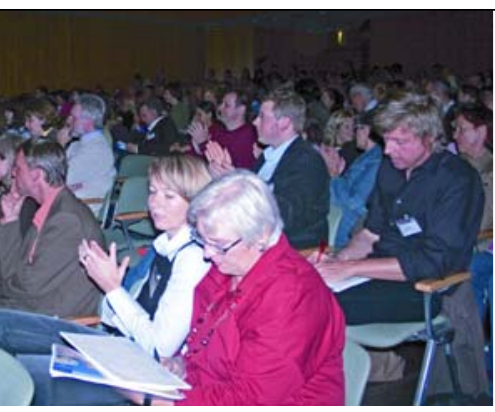

Abb. 1 Viel Applaus gab es auf der Eröffnungsveranstaltung.

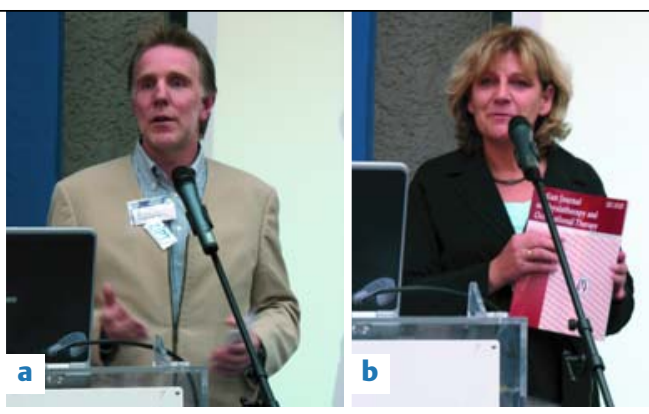

Abb. 2 a und b Uwe Harste und Rosi Haarer-Becker begrüßten die Besucher des Kongresses.

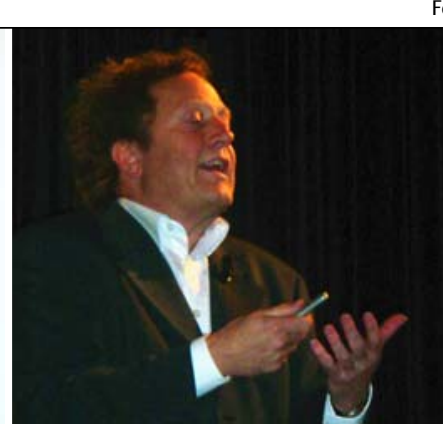

Abb. 3 Theo Mulder zog die Zuhörer in seinen Bann.
Fotos: E. Baumann, F. Klenger, I. Grobe

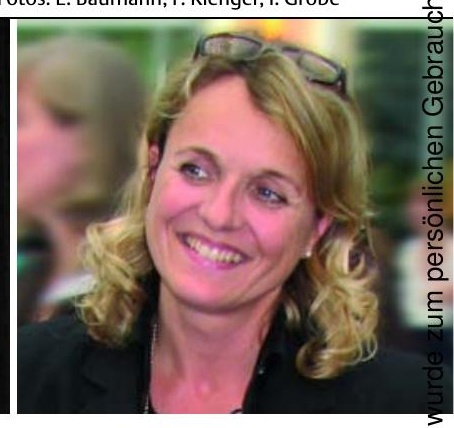

Abb. 4 Annette Probst präsentierte ihr neues Modell. ternehmerisch denken und handeln" Informationen und Anregungen.

Bewegung: das zentrale Element • Schon bei der Eröffnungsveranstaltung spürten die Besucher das hohe Niveau des Kongresses: Zwei Experten für Bewegungswissenschaft beleuchteten das zentrale Element Bewegung aus unterschiedlichen wissenschaftlichen Perspektiven. Der niederländische Neuropsychologe Theo Mulder (॰ Abb. 3) erläuterte die adaptiven Fähigkeiten des tennisschläger in die Hand, schlug er den Ball flink und wie ein Profi in die Luft. Der behandelnde Therapeut wusste, dass er in jüngeren Jahren leidenschaftlich Tischtennis spielte. Durch den emotionalen Bezug zum Sportgerät konnte die alte kortikale Repräsentation dieser Bewegung aktiviert werden. Fazit: Emotional positiv gespeicherte Bewegungserfahrungen fördern motorisches Lernen.

Neues Modell der menschlichen Bewegung Ganz anders näherte sich die Bewe-
Sie ist durch sozialen Sinn sowie Emotionalität gekennzeichnet, tritt in einem bestimmten Kontext auf, und ihr Ausdruck, ihre Bedeutung kann von Kultur zu Kultur unterschiedlich sein und ist historisch veränderbar. In dem von ihr entwickelten Modell überträgt Probst diese Erkenntnisse auf die Bedeutung von menschlicher Bewegung in der Physiotherapie. Das Modell wird in der nächsten September-Ausgabe der Fachzeitschrift physioscience veröffentlicht. Damit eine physiotherapeutische Behandlung ge- 
lingen kann, betonte die Professorin am Ende ihres Vortrags, gilt es mit dem Patienten gemeinsam Sinn, Lebensweltbezug und Alltagsrelevanz von Bewegungshandlungen herzustellen.

Die neue Fortbildungsmöglichkeit: CPTE • „Ich erhoffe mir durch den Besuch hier auf dem Kongress neuen Input für meinen physiotherapeutischen Alltag, außerdem finde ich es toll, so viele Gleichgesinnte auf einem Haufen zu treffen“, beschrieb eine Physiotherapeutin aus Hannover den Grund ihrer Anreise. Für den Kongressbesuch konnte sie auch Fortbildungspunkte erlangen. Fortbildung spielt in der Physiotherapie eine große Rolle und ist seit kurzem gesetzlich Pflicht. Meist bilden sich Therapeuten in Kursen fort. Eine neue Möglichkeit der Fortbildung entwickelten die FH Osnabrück, der Bundesverband selbstständiger Physiotherapeuten IFK und der Georg Thieme Verlag. Sie stellten auf dem Kongress die ersten Ergebnisse ihres Projekts IQ Phys (Instrumente für Qualitätssicherung in der Physiotherapie) vor. Dahinter steckt die Idee der kontinuierlichen Fortbildung - Continuing Physiotherapy Education (CPTE). Die Gruppe hat Qualitätskriterien für sogenannte CPTE-Artikel entwickelt (zum Beispiel physiopraxis.Refresher aus physiopraxis 5/07), die am Ende einen Bogen mit zehn Multiple-Choice-Fragen zur Prüfung des Lernerfolges haben. Diesen kann der Teilnehmer ausgefüllt an den IFK bzw. den Georg Thieme Verlag senden. Hat er die Mindestpunktzahl erreicht, kann er etwas gewinnen. Das Ziel des Projektes ist es aber, die richtigen Antworten mit Fortbildungspunkten zu honorieren. So könnten sich Physiotherapeuten auch von zu Hause fortbilden und Punkte sammeln, um innerhalb von vier Jahren auf die vorgeschriebenen 60 Fortbildungspunkte zu kommen. Doch bis das so weit ist, sind noch einige Hürden zu überspringen.

Ganganalyse ist trainierte Geschicklichkeit Preise konnten auch Teilnehmer des Seminars zum Thema Ganganalyse von Kirsten Götz-Neumann gewinnen. Die Physiotherapeutin machte auf anschauliche Weise klar, dass voreilige Schlüsse bei der Ganganalyse kontraindiziert sind. Vor Beginn ihres Seminars hatte sie unter Ausschluss der Öffentlichkeit zwei Freiwilligen Tapestreifen aufgeklebt, um die Gelenkbeweglichkeit zu fixieren. Die Aufgabe an das Publikum lautete: „Schaut euch das Gangbild an und sagt, wie viele Gelenke an der unteren Extremität fixiert sind!“ Die erste Probandin watschelte im Entengang sichtlich mühsam den Gang auf und ab. Der zweite Proband lief, als hätte er an beiden Beinen lange Stöcke gebunden. Bei der Abstimmung mittels roter und grüner Stimmkarten wurde schnell klar: Die visuelle Ganganalyse führt bei ungeübten Augen zu einer hohen Fehlerquote! Die Lösung war für alle verblüffend: Bei der ersten Probandin war lediglich das rechte Sprunggelenk in Dorsalextension fixiert, während beim zweiten Probanden das fixierende Tape auf der Lendenwirbelsäule klebte. Die aus Düsseldorf stammende Physiotherapeutin machte

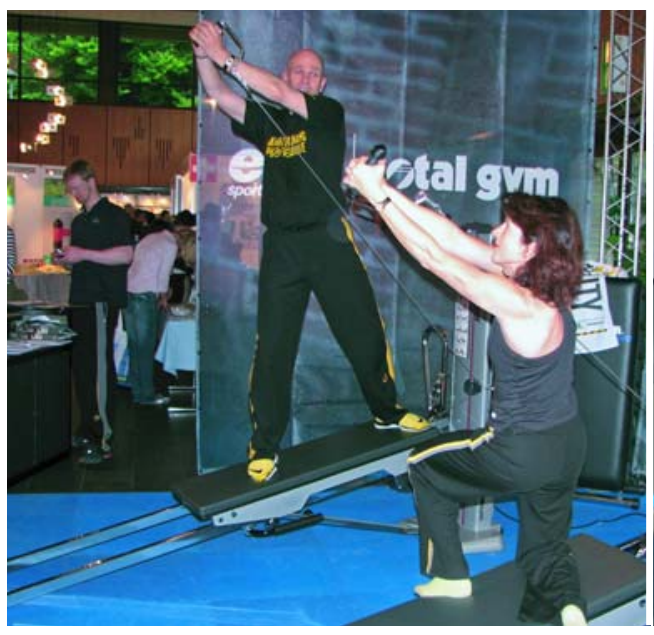

Abb. 5 Geräte vor Ort testen, das konnten die Besucher auf der Industrieausstellung.

dem Publikum mit ihrer rheinischen Art Mut: „Die beobachtende Ganganalyse ist trainierte Geschicklichkeit."

Begeistert von dem Seminar war auch Mathilde Harms, Schulleiterin der Professor Grewe Schule in Osnabrück. Sie baut neben Beinachsentraining aus der Spiraldynamik auch die Ganganalyse in ihren Unterricht mit ein: „Ich finde es wichtig, up to date zu bleiben und meinen Schülern darin einen Einblick zu geben. Die Schulen heutzutage kommen nicht mehr umhin, sich mit der Wissenschaft in der Physiotherapie auseinanderzusetzen“, sagte Harms, die vom Kongress mit vielen Anregungen nach Hause fuhr.

\section{Service und Kommunikation als Marke-} tinginstrument • Gut besucht waren auch die Seminare zum Themenschwerpunkt „Unternehmerisch denken und handeln“. Hier bekamen Selbstständige oder die, die es einmal werden wollen, Informationen und Tipps, um ihre Physiotherapiepraxis für den zunehmenden Wettbewerb fit zu machen.

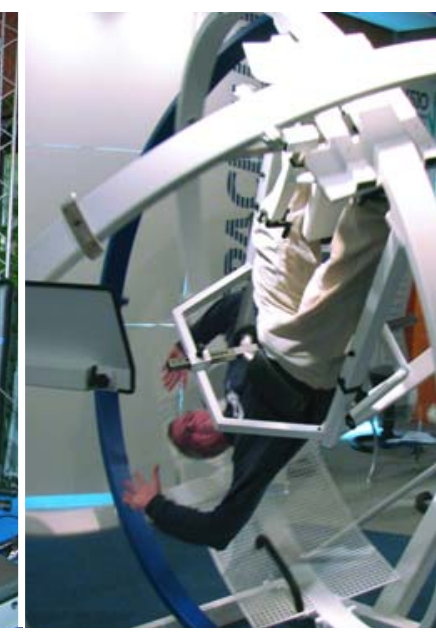

Abb. 6 Der Spacecurl ermöglicht ein Training in drei Dimensionen.

„Achtzig Prozent des Erfolgs einer Praxis hängen vom Service ab!“, erklärte Unternehmensberater Ralf Buchner in seinem Seminar. Er versuchte die Teilnehmer lebhaft davon zu überzeugen, dass es wichtig sei, außer in fachliche Fortbildungen auch in die Praxisausstattung, Sauberkeit sowie Freundlichkeit an der Rezeption zu investieren.

Ideen für neue Möglichkeiten und spannende Herausforderungen für die Physiotherapie bekamen die Teilnehmer des Seminars „Wirtschaftlichkeit und Menschlichkeit“. Physiotherapeutin Tanja Latour sprach darin

über die Kommunikationsfähigkeit. Die Referentin stellte fest: „Die Sprache und Wortwahl ist der wichtigste Faktor für das Selbstmarketing!“ Ihrer Meinung nach sollte man nicht nur in physiotherapeutische Techniken investieren, sondern auch Kommunikationskurse besuchen.

Zukunft: Physiotherapeuten als Therapeuten und Berater $\gg$ In der abschließenden Diskussionsveranstaltung am Samstag ging es um die Frage „Wo stehen die Einzelpraxen im Jahre 2015?" Unter der Leitung des Gesundheitsökonomen und Logopäden JensPeter Claußen entstand eine lebhafte und inhaltsvolle Diskussion zwischen den Experten mit therapeutischer und kaufmännischer Markterfahrung und dem Publikum. Cornelia Schneider stellte unter anderem die Frage in den Raum: „Wo sind wir Physios mit unserer Beratungskompetenz? Die meisten Kosten im Gesundheitssystem in Deutschland verursachen Muskel- und Skeletterkrankungen. Wer sind 2015 dafür die Experten, wenn 
nicht wir?" Ein freiberuflich tätiger Physiotherapeut meinte darauf, dass zu viel Beratung für ihn nichts mehr mit dem Kernjob der physiotherapeutischen Behandlung zu tun habe. Und damit sprach er sicherlich vielen Praktikern aus der Seele. Doch die Experten waren sich einig, dass ohne Marketingstrategien wie Imagepflege, Beratung, Service ein Großteil der Praxen verloren gehen wird.

Auch Haftungsfragen kamen zur Sprache: „Ein Patient, der keine Verordnung mehr bekommt, möchte weitere Behandlungen als Hausbesuche privat weiter finanzieren.

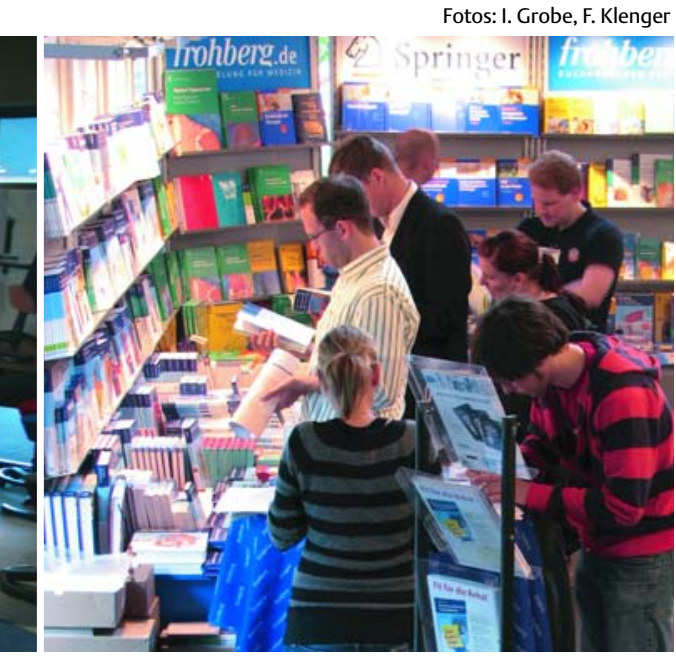

Abb. 7 Frischgedruckt und altbewährt. Am Bücherstand konnte man probelesen.

Wie ist hier die Rechtslage?“, fragte ein Teilnehmer. Der Unternehmensberater Ralf Buchner erklärte daraufhin, dass er diese Behandlung zu Hause so nicht machen dürfe, da seine Zulassung als Therapeut an die Praxis gebunden sei. Buchner schlug vor: „Machen Sie mit einem ortsansässigen Taxiunternehmen einen Deal und vereinbaren Sie einen Shuttle-Service, der die Patienten zu Ihnen in die Praxis bringt!“

Obwohl sich klar abzeichnetet, dass der Gesundheitsmarkt ein hart umkämpfter ist und sich Lohndumping mehr und mehr breitmacht, waren die Beiträge konstruktiv, und auf der Veranstaltung wurde nicht gejammert, wie Claußen in seinem Abschlusswort zufrieden feststellte.

Ideen sammeln auf dem Markt der Möglichkeiten • Anregungen erhielten die Besu응 cher in den Pausen auch auf dem Markt der Möglichkeiten, der begleitenden Industrieausstellung des physiokongresses. „Jetzt muss ich nur noch schnell mein Examen hin- ter mich bringen, dann werde ich auch einen Meditaping-Kurs machen“, erzählte eine Schülerin an einem Tape-Stand begeistert. Kurze Zeit vorher klebte Dieter Sielmann einem ihrer Klassenkameraden, der über tiefe LWS-Schmerzen klagte, einen Tapestreifen quer auf den Unterbauch sowie auf die Lendenwirbelsäule. Und siehe da, beim erneuten Bücken fühlte sich der Rücken gleich besser an. „Das Interesse ist groß“, berichtet Sielmann. „Fast alle kennen zwar Kinesiotape, doch viele wollen es auch mal an sich selbst ausprobieren und spüren, um dann endgültig überzeugt zu sein. " Er ist schon zum dritten Mal auf dem physiokongress vertreten und verpasst jedem bereitwillig ein buntes Tape.

Dass Physiotherapeuten eine Affinität zur Bewegung haben, wurde beim Rundgang über den Markt der Möglichkeiten deutlich. An interaktiven Ständen der insgesamt über 60 Aussteller wurden die Geräte getestet (॰ Abb. 5). Ob es ums Ausprobieren von Trampolinen, Kippbrettern oder anderen Gymnastikutensilien ging oder um das Erleben einer neuen Körpererfahrung wie zum Beispiel das dreidimensionale Bewegen im Spacecurl (॰ Abb. 6) oder dem alternierenden bzw. pulsierenden Vibrationstraining. Neben Informationen über Fortbildungen und Berufsverbände konnten die Kongressbesucher auf dem Markt der Möglichkeiten auch in Ruhe in Büchern stöbern ( $\odot$ Abb. 7).

COPD: gesamte Muskulatur trainieren Eine ansehnliche Themenvielfalt bot auch der Schwerpunkt „Sport und Physiotherapie“: von Sportphysiotherapie bis hin zu Sport und Krafttraining in der Physiotherapie. Geert Jeuring, der sich auf die Physitherapie der chronisch obstruktiven Lungenerkrankung (COPD) spezialisiert hat, referierte zu diesem Thema: „Die Atemtherapie hat einen hohen Stellenwert bei der Behandlung von Patienten mit COPD. Es hat sich jedoch gezeigt, dass sie das Fortschreiten der Krankheit nicht bremst und das Alltagsleben nicht ausreichend normalisieren kann“, betonte Jeuring. Auf die gesamte Muskulatur sollte sich das Augenmerk verstärkt richten, um den typischen Teufelskreis zu durchbrechen, der mit Atemnot und Angst vor Anstrengung beginnt und über fehlende Muskelbeanspruchung und Muskelabbau immer tiefer in die Inaktivität und soziale Isolation führt. Laut Studien lassen sich mit einem kombinierten Kraftausdauertrainings Folgen der COPD wirksam bekämpfen und dürfen daher bei der Behandlung auf keinem Fall fehlen. 
